\title{
Performance Analysis for Capacitive Electrical Neural Interfaces
}

\author{
C. Koca, E. Dinc, H. Ramezani, O. B. Akan \\ Internet of Everything Group, Department of Engineering, University of Cambridge, UK, CB3 0FA. \\ E-mails: $\{$ ck542, ed502, hr404, oba21\}@cam.ac.uk
}

\begin{abstract}
Neural interfaces will pave the way for novel treatment methods for neural disorders, which are due to communication problems in nervous system. Such disorders include spinal cord injuries, Alzheimer's and Multiple Sclerosis. In this work, we present a novel neural stimulator, which will act as the transmitter part of a neural interface. We perform in detail physical analysis of such a device for the first time, considering the electrostatic and capacitive effects. We also establish the stimulation requirements of the post-synaptic neuron and support our findings with COMSOL simulations. This work will pave the way to the design of more efficient neural stimulators.
\end{abstract}

\section{INTRODUCTION}

Monitoring local neuronal activity is becoming increasingly prevalent, paving way for novel treatment methods for neurological disorders such as spinal cord injury and neural interface for artificial limbs. Towards this purpose, electrophysiological recording techniques have attracted significant attention with their high temporal resolution, but lacking spatial resolution. To tackle this problem, different high density electrode arrays have been developed and tested $[3,4]$.

For high density electrode arrays, there are three main methods to interface with neurons: (1) chemical stimulation via injecting neurotransmitters, (2) optical stimulation by placing genetically modified photo-active proteins (optogenetics) in neurons, (3) electrical stimulation via applying faradaic current or capacitive electric field (CES). While chemical and optical stimulation require altering physiological content of target cells, electrical stimulation can be performed without creating changes in the medium. In electrical stimulation, faradaic interfacing with the medium is undesirable due to increasing reaction probability on electrode surface and proteins in the cerebrospinal fluid (CSF). Therefore, electrode arrays are passivated with dielectric materials such that applied potential creates an electric field and potential difference are created by the movement of ions in CSF. Although passivated high density electrodes are tested for sensing neural signals from individual neurons, theoretical limitations of this method in stimulation is untouched.

In this paper, we propose a theoretical model and simulation environment in COMSOL Multiphysics to determine performance and limitations of CES for the first time in the literature. By using the proposed approach, we demonstrate the feasibility of stimulating neurons via CES and determine critical design parameters such as achievable stimulation frequency, range and electrode dimensions. Therefore, this paper provides guidelines for future neural interfaces.

The remainder of the paper is organised as follows. Section I includes the excitability threshold of neurons. Section II and III contain the theoretical analysis and simulation environment for capacitive electrical stimulation, respectively. Simulation results are presented in Section IV. The conclusions are discussed in Section V.

\section{STIMUlation OF NEURON}

Nervous system transmits information in the form of electrical signals, i.e. action potentials, which is all-or-none process. If a neuron stimulated above a certain threshold, the neuron will fire an action potential and stimulate all dendrites that are connected. Learning and information processing take place in synapses whose excitability threshold shows shortterm and long-term variability, also known as plasticity. In addition, physiological conditions such as distance from soma and neuron type have a direct effect on the threshold [1].

Neural spikes are generally generated via voltage-gated sodium channels [2]. These channels slowly depolarise the cell membrane up to a certain threshold. Any stimulation exceeding this threshold causes a positive feedback, which results in an instability causing neighbouring sodium channels to open and this process ends up with an action potential. For CES, the critical point is exceeding this certain threshold to initiate an action potential. [1] presents an empirical model to estimate dynamic threshold for excitability of neurons. According to experimental observations and [1], excitability threshold of neuron is generally $20-30 \mathrm{mV}$ higher than resting potential of neuron membrane, which is $-70 \mathrm{mV}$ on average. Thus, the excitability threshold to create an action potential, in this paper, is assumed as $30 \mathrm{mV}$ for the sake of simplicity.

\section{Simulation EnVironment}

Electrical double layer (EDL) is an important phenomenon to describe surface interaction between electrolyte and electrode surface. EDL means two charge layers in the vicinity of electrode surface as shown in Fig. 1. The first layer is caused by the charges on the electrode that have opposite sign. The second layer is formed due to the Coulomb force and electrically screen the first layer. According to the GouyChapman model (GCM), the charge distributions can be predicted with Maxwell-Boltzmann statistics, and decreases exponentially away from electrode with the rate of Debye length that can be calculated as

$$
x_{D}=\sqrt{R T \varepsilon_{\text {bulk }} \varepsilon_{0} /\left(2 F^{2} c_{\text {bulk }}\right)},
$$


where $R$ is the gas constant, $T$ is the temperature in $\mathrm{K}, \varepsilon_{0}$ is the

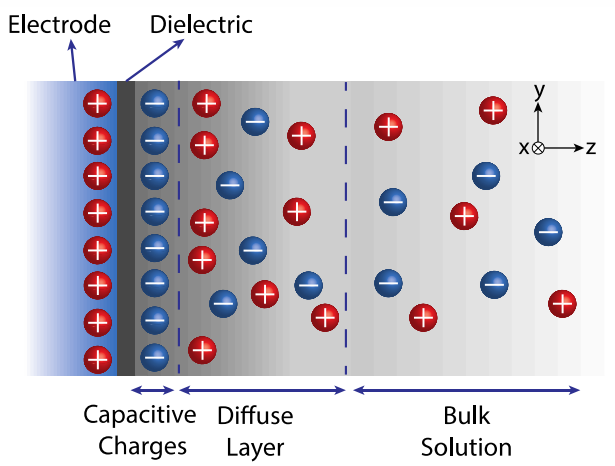

Fig. 1. Schematic representation of a double layer on CES.

permittivity of the free space, $\varepsilon_{\text {bulk }}$ is the dielectric constant of the electrolyte, $F$ is the Faraday constant, and $c_{\text {bulk }}$ is the molar concentration of the electrolyte. In this way, the potential of the medium decreases exponentially and becomes approximately constant in the bulk solution in the steady state conditions (This requires certain time.). The Stern modification to GCM considers the plane closest to the electrode, which is one atom thick and have opposite charge to the surface of the electrode. The effect of this layer can be modelled as a Stern capacitance with constant dielectric of the electrolyte and Stern thickness of $x_{s}$ (assumed as $0.5 \mathrm{~nm}$, in this paper). In CES, there is an additional capacitance due to the passivation of electrode surface. The dielectrics capacitance is in series with the Stern capacitance such that the total capacitance per area of the system can be calculated as

$$
C_{D L}=\frac{C_{\text {Stern }} C_{\text {die }}}{C_{\text {stern }}+C_{\text {die }}}=\frac{\varepsilon_{\text {bulk }} \varepsilon_{P M M A} \varepsilon_{0}}{x_{S} \varepsilon_{P M M A}+\varepsilon_{\text {bulk }} x_{P M M A}},
$$

where $\varepsilon_{P M M A}$ is the dielectric constant of PMMA, and $x_{P M M A}$ is the length of the dielectric layer. Therefore, the electrode boundary is controlled by the following equation

$$
\rho_{\text {surf }}=\left(\varphi_{\text {Electrode }}-\varphi_{\text {Electrolyte }}\right) C_{D L}
$$

where $\varphi_{\text {Electrode }}$ is the applied potential, and $\varphi_{\text {Electrolyte }}$ is the potential of the electrolyte that can be described by Poisson equation. The electrolyte movement in the diffuse layer is described by diffusion in the presence of electrostatic forces. To this end, the Nernst-Planck equation is utilised to calculate the charge distribution in electrolyte, and this equation can be represented as

$$
J_{i}=-D_{i} \nabla C_{i}-\frac{D z e}{k_{B} T} C \nabla \varphi,
$$

where $J_{i}$ is the ion flux in $\mathrm{mol} /\left(\mathrm{m}^{2} . \mathrm{s}\right)$, and $D_{i}$ is the diffusion coefficient in $\mathrm{m}^{2} / \mathrm{s}, \mathrm{C}$ is the concentration in $\mathrm{mol} / \mathrm{m}^{3}, z$ is the valance charge, $e$ is the electron charge, $k_{B}$ is the Boltzmann constant.

In the COMSOL simulations, we model the electrolyte having positive and negative ions. For the sake of simplicity, ions are assumed to have the same concentration in the electrolyte with the same diffusion coefficients. Potential applied through the metal electrode with respect to the right of the container which is assumed as ground having open boundary such that positive and negative ions always have equal concentrations. Other three boundaries (bottom, left and top) are assumed as closed boundaries with no concentration flux. At the end, we monitor the total charge $\rho=C_{+}+C_{-}$, where $C_{+}$and $C_{-}$stand for positive and negative ions, and voltage changes in the medium to predict the performance of CES.

\section{ThEORETICAL ANALySIS}

In this section, we analyse the generation of the required potential difference to stimulate the neuron. The induction of potential difference can be investigated in two parts: Instant electrostatic induction, and charge accumulation.

\section{A. Instant Electrostatic Induction}

When a potential difference is applied to the conducting plate, the potential difference instantly effects its surroundings. Since the net charge distribution in the vicinity of the stimulator is zero, the system can be described with a boundary problem:

$$
\begin{gathered}
\varphi=V_{0}, \text { in } 0<x<x_{l}, 0<y<y_{l}, z=0 \\
\varphi=0, \text { elsewhere. }
\end{gathered}
$$

where the target is at $\left(x_{l} / 2, y_{l} / 2, z\right)$, i.e., directly above the stimulator at a distance $z$. In order to solve this problem, we look for potential functions of product form such that we can use separation of variables, i.e.,

$$
\varphi(\mathrm{x}, \mathrm{y}, \mathrm{z})=\mathrm{X}(\mathrm{x}) \mathrm{Y}(\mathrm{y}) \mathrm{Z}(\mathrm{z}),
$$

and the Laplace Equation becomes

$$
\frac{1}{X} \frac{d^{2} X}{d x^{2}}+\frac{1}{Y} \frac{d^{2} Y}{d y^{2}}+\frac{1}{Z} \frac{d^{2} Z}{d z^{2}}=0
$$

Solution to (8) is obtained by assigning all parts of (8) to a constant such that the summation of the constants is equal to zero, i.e.,

$$
\begin{aligned}
& \frac{1}{X} \frac{d^{2} X}{d x^{2}}=C_{x}, \\
& \frac{1}{Y} \frac{d^{2} Y}{d y^{2}}=C_{y}, \\
& \frac{1}{Z} \frac{d^{2} Z}{d z^{2}}=C_{z} .
\end{aligned}
$$

We solve (5-7) by choosing $C_{x}=-k^{2}, C_{y}=-l^{2}$. As a result, $C_{z}=k^{2}+l^{2}$, and the solutions to (5-7) becomes

$$
\begin{gathered}
\mathrm{X}(\mathrm{x})=\mathrm{A} \sin k x+B \cos k x, \\
\mathrm{Y}(\mathrm{y})=\mathrm{C} \sin l y+D \cos l y, \\
\mathrm{Z}(\mathrm{z})=\mathrm{E} e^{\sqrt{k^{2}+l^{2}} z}+F e^{-\sqrt{k^{2}+l^{2}} z} .
\end{gathered}
$$

In order to solve (12-14), we assume that the voltage change due to the stimulator only occurs within the region closed by semi-infinite rectangular prism with base on the $x-y$ plane bounded by $0<x<x_{l}, 0<y<y_{l}$ and voltage is for $\mathrm{z} \rightarrow \infty$. Note that these assumptions are perfectly valid for near-fields, i.e., $\mathrm{z} \ll x_{l}, y_{l}$. Hence, the constants B, D and $\mathrm{E}$ vanishes and 
$\mathrm{k}=n \pi / x_{l}, \mathrm{l}=m \pi / \mathrm{y}_{l}$ and the form of the general solution for the boundary problem becomes

$$
\begin{aligned}
\varphi_{m, n}=F_{m, n} e^{-\pi z \sqrt{\left(n / x_{l}\right)^{2}+\left(m / y_{l}\right)^{2}}} & \\
& \times \sin \left(n \pi x / x_{l}\right) \sin \left(m \pi y / y_{l}\right) .
\end{aligned}
$$

where $F_{n, m}$ is a constant depending on the applied voltage $V_{0}$ and $\mathrm{n}, \mathrm{m}$.

$F_{m, n}$ coefficients can be obtained using (15) and using the orthogonality of $\sin \left(n \pi x / x_{l}\right)$ and $\sin \left(m \pi x / y_{l}\right)$ with $\sin \left(n^{\prime} \pi x / x_{l}\right)$ and $\sin \left(m^{\prime} \pi x / y_{l}\right)$. The final result with applied $V_{0}$ then becomes

$$
\begin{aligned}
\varphi_{V_{0}}(x, y, z)= & \sum_{n=1}^{\infty} \sum_{m=1}^{\infty} \frac{16 V_{0}}{\pi^{2} n m} e^{-\pi z \sqrt{\left(n / x_{l}\right)^{2}+\left(m / y_{l}\right)^{2}}} \\
& \times \sin \left(n \pi x / x_{l}\right) \sin \left(m \pi y / y_{l}\right) \text { for odd } \mathrm{n}, \mathrm{m}
\end{aligned}
$$

\section{B. Charge Accumulation}

Due to the potential difference generated by applying a voltage on the plate, the free charges in the vicinity move close to the plate. Therefore, the electrode forms a capacitor where the PMMA coating acts as a dielectric. Since the amount charge accumulated in the capacitor is the time integral of current we find that

$$
\mathrm{Q}(\mathrm{t})=\sum_{i} c_{i}(x, y, z) z_{i} \mu_{i} x_{l} y_{l} \int_{0}^{t} E(x, y, z, t) d t^{\prime},
$$

where $c_{i}(x, y, z)$ is the concentration, $z_{i}$ is the charge, and $\mu_{i}$ is the charge mobility for a certain ion type. Since (17) is very hard to solve as it is, we make several assumptions:

1. The charge density is considered constant.

2. $E(x, y, z, t)$ does not depend on $\mathrm{x}$ and $\mathrm{y}$.

3. $E(x, y, z, t)$ is constant for $\Delta \mathrm{z}$.

4. The movement of ions is largely influenced by $\mathrm{E}$, rather than concentration gradient.

Note that 1 and 4 are very similar and hold accurately as long as the charge accumulated on the capacitor is negligible compared to the charge concentration. 2 only negates the currents in the $\mathrm{x}$ and $\mathrm{y}$ directions which are simply oscillations in the harmonics constituting (12), which vanish for large $m$ and n. Finally, 3 is a valid assumption for short $\Delta \mathrm{z}$.

Under these assumptions, the voltage on the capacitor becomes

$$
\begin{aligned}
& V_{C}(t)=\frac{Q(t)}{C}=\frac{\sum_{i} c_{i} z_{i} \mu_{i} x_{l} y_{l} \int_{0}^{t} E(t) d t^{\prime}}{\varepsilon_{0} \varepsilon_{r} \frac{x_{l} y_{l}}{d}}, \\
& V_{C}(t)=\frac{d}{\varepsilon_{0} \varepsilon_{r}} \sum_{i} c_{i} z_{i} \mu_{i} \int_{0}^{t} E(t) d t^{\prime},
\end{aligned}
$$

where $d$ is the thickness of the dielectric and $\varepsilon_{r}$ is the relative permittivity of the PMMA. Hence, the potential becomes

$$
\varphi(\mathrm{t})=\varphi_{\left(V_{0}-V_{c}\right)}(t)+\varphi_{\text {dipole }}(\mathrm{t}),
$$

where the first term is calculated by substituting $V_{0}-V_{c}$ instead of $V_{0}$ in (12) and the second term is the dipole potential due to the charges on the capacitor given by

$$
\varphi_{\text {dipole }}(\mathrm{x}, \mathrm{y}, \mathrm{z}, \mathrm{t})=-\frac{z d Q(t)}{4 \pi \varepsilon_{0} x_{l} y_{l}} \int_{0}^{x_{l}} \int_{0}^{y_{l}} \frac{d x^{\prime} d y^{\prime}}{\left[\left(x-x^{\prime}\right)^{2}+\left(y-y^{\prime}\right)^{2}+z^{2}\right]^{3 / 2}} .
$$

\section{NUMERICAL RESULTS}

In this section, we present numerical results for our analysis and simulation. A potential difference of $30 \mathrm{mV}$ is enough for generating action potential.

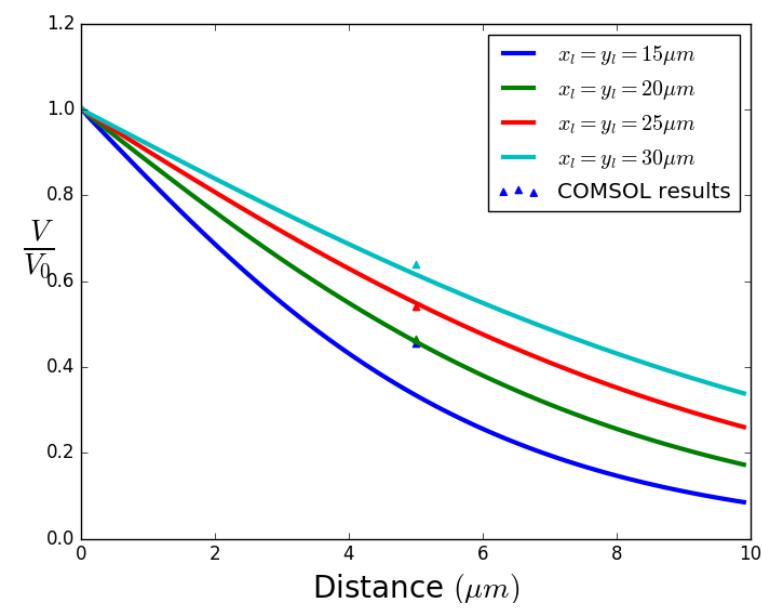

Fig. 2. Induced voltage ratio for plates of different sizes vs distance from the centre of the stimulator.

First, we utilised the analytical model to calculate the excitation limit unburdened by ion movements, i.e. instantaneously rising voltage. As we can see in Fig. 2, the induced voltage drops linearly with distance from the centre of the stimulator. Since a voltage of $30 \mathrm{mV}$ is necessary for stimulation, assuming $5 \mu \mathrm{m}$ distance from the center of the stimulator to the neuron, $z_{0}$, even applying $70 \mathrm{mV}$ is enough if the simulator dimensions are $20 \mu \mathrm{m} \times 20 \mu \mathrm{m}$. The COMSOL results for instantaneous induction is calculated by using very low rise time $(0.1 \mathrm{us})$.

According to our results, the simulated CES can stimulate a neuron even applying $70 \mathrm{mV}$. However, instantaneous rising voltage is not realisable in the real-world scenarios. Hence, we use a finite rise time in COMSOL simulations. The maximum percent difference between our theoretical analysis and simulations is $26.53 \%$ for $15 \mu \mathrm{m} \times 15 \mu \mathrm{m}$ stimulator. This is most probably due to our assumptions not holding perfectly for smaller size plates. The rest of the results fit our theoretical analysis quite well, with error values between $1.61 \%$ for $20 \mu \mathrm{m} \times 20 \mu \mathrm{m}, 1.71 \%$ for $25 \mu \mathrm{m} \times 25 \mu \mathrm{m}$ and $3.74 \%$ for $25 \mu \mathrm{m} \times 25 \mu \mathrm{m}$ plates. 
Since this device is to be implanted in body, the distance and orientation may not be adjusted perfectly. We can calculate the potential in case the centre is shifted $x_{0}$ and $y_{0}$, we can easily use (12) to calculate the potential at $\varphi\left(x_{l} / 2-\right.$ $\left.x_{0}, y_{l} / 2-y_{0}, z_{0}\right)$. The percent change due to the deviations in $\mathrm{x}$ direction for a target at $10 \mu \mathrm{m}$ is given in Fig. 3. As we can see in Fig 3, even for small size stimulators, the percent difference is reasonable and manageable. Note that a $50 \%$ extra attenuation due to orientation and distance deviations can be negated by increasing the applied voltage two folds.

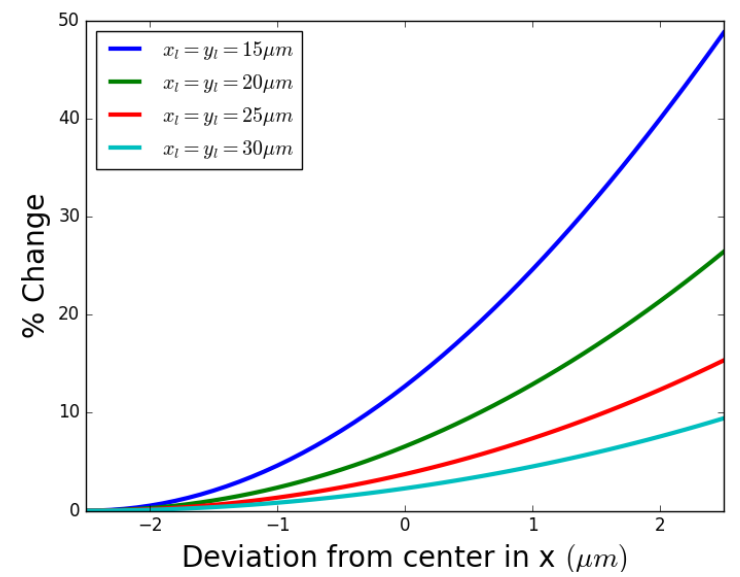

Fig. 3. Percent potential change at the target due to the deviations in the $\mathrm{x}$ direction

In case the deviation is not in the orientation of the stimulator with respect to the target but in the distance to the target, we can use a similar approach to calculate the percent potential change at the target. We present the effects of small deviations from the intended $10 \mu \mathrm{m}$ target distance for different stimulator sizes in Fig. 4. As you can see in Fig. 4, small deviations for tolerable, even for small stimulators.

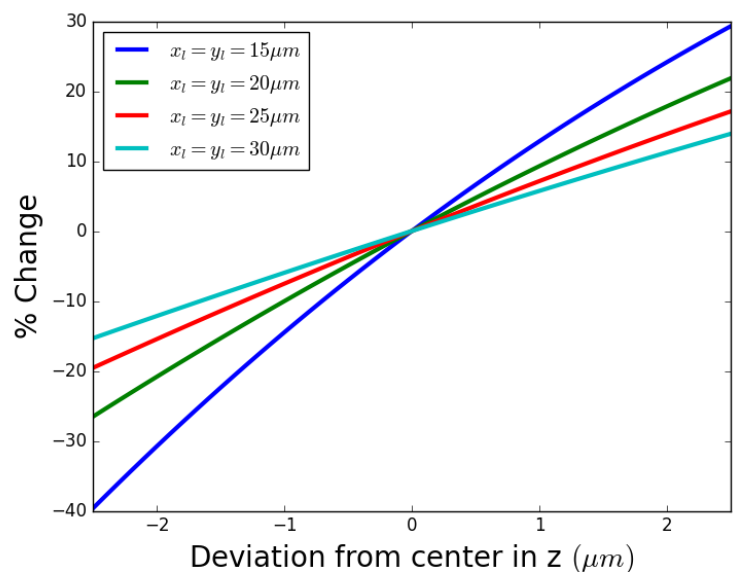

Fig. 4. Percent potential change at the target due to the deviations in the $\mathrm{z}$ direction

In COMSOL simulations, potential difference at the target point is created by the movement of ions as explained in Section III. We applied a gradually rising potential difference of one volt at the electrode at $1 \mathrm{~ms}$ in order to analyse the transient response of the neural interface. As seen Fig. 5, decreasing rise time significantly increases the maximum potential difference generated on the target neuron. In addition, the duration of stimulation is increasing with the increasing rise time at the expense of maximum generated voltage. Hence, there is a significant trade-off between applied voltage and stimulation duration.

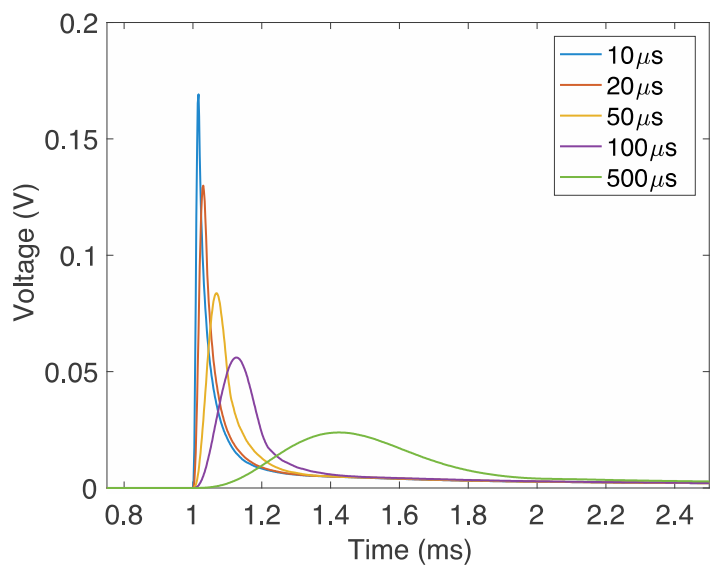

Fig. 5 Induced Potential at the target for different rise times

\section{CONCLUSION}

In this work, we have calculated the exact potential due to a capacitive electrical neural interface at a target. We have demonstrated that such devices are capable of inducing the necessary potential difference for neural stimulation. We have also shown that capacitive electrical interfaces can tolerate the small deviations in their operation medium. We also started analysing the factors that will affect the stimulation period, such as charging of the capacitor.

In our future works, we will fully analyse the charging of the capacitor and its effects in the rate of change of voltage and fully demonstrate the excitability of neurons using such a device. We will also calculate the energy per use and maximum operating frequency of such a device.

\section{ACKNOWLEDGMENT}

This work was supported in part by the ERC project MINERVA (ERC-2013-CoG \#616922), and the ERC Project MINERGRACE (ERC-2018-PoC \#780645).

\section{REFERENCES}

[1] J. Platkiewicz, R. Brette. "A threshold equation for action potential initiation." PLoS computational biology 6.7, 2010.

[2] B. Hille, Ion channels of excitable membranes, vol. 507. Sunderland, MA: Sinauer, 2001.

[3] Duygu Kuzum et al. "Transparent, Flexible, Low Noise Graphene Electrodes for Simultaneous Electrophysiology and Neuroimaging," Nat Commun; 5: 5259. doi:10.1038/ncomms6259.

[4] W. Gong et al. "Multiple Single-Unit Long-Term Tracking on Organotypic Hippocampal Slices Using High-Density Microelectrode Arrays," Frontiers in Neuroscience, vol. 10, pp. 537, 2016. 